\title{
Three-Dimensional Unsteady State Temperature Distribution of Thin Rectangular Plate with Moving Point Heat Source
}

\author{
Yogita M. Ahire ${ }^{1}$ and Kirtiwant P. Ghadle ${ }^{2}$ \\ ${ }^{1}$ Department of Applied Science, PVG'S College of Engineering, Nashik, Maharashtra, India \\ ${ }^{2}$ Department of Mathematics, Dr. Babasaheb Ambedkar Marathwada University, Aurangabad 431004, India \\ Correspondence should be addressed to Kirtiwant P. Ghadle; drkp.ghadle@gmail.com
}

Received 30 March 2016; Accepted 20 July 2016

Academic Editor: Dnyaneshwar S. Patil

Copyright (c) 2016 Y. M. Ahire and K. P. Ghadle. This is an open access article distributed under the Creative Commons Attribution License, which permits unrestricted use, distribution, and reproduction in any medium, provided the original work is properly cited.

\begin{abstract}
This paper deals with the study of thermal stresses in thin rectangular plate subjected to point heat source which changes its place along $x$-axis. Governing heat conduction equation has been solved by using integral transform technique. Results are obtained in the form of infinite series. As a special case, aluminum plate has been considered and results for thermal stresses have been computed numerically and graphically.
\end{abstract}

\section{Introduction}

Material properties are dependent on change in temperature. The properties like elasticity and stresses at various temperatures have been studied. These nonisothermal problems of theory of elasticity have attracted the attention of many. The temperature dependent properties are focused on various fields like aerodynamics heating which produces intense thermal stresses reducing the strength of structure of high velocity aircraft [1]. Steady state thermal stresses with axis symmetric temperature distribution in a circular plate subjected to the upper surface with respect to zero temperature on the lower surface and thermally insulated circular edge have been determined by [2]. On fixed and simply supported edges [3] has calculated thermal deflection of associated axis symmetrically heated circular plate. Reference [4] has considered quasi-static thermal stresses in a thin circular plate due to transient temperature applied along the edge of a circle on the upper face with respect to lower face at zero temperature and a thermally insulated fixed circular edge. Reference [5] studied an inverse unsteady state thermoelastic problem of a thin rectangular plate. Quasi-static thermoelastic problem of an infinitely long circular cylinder has been calculated by [6]. Temperature distribution, thermal functions, and displacement at any point of semi-infinite rectangular slab with internal heat source using integral transform technique are solved by [7]. Using integral transform technique and Green's theorem [8] has determined temperature distribution and thermal stresses by taking second kind boundary condition in thin rectangular plate with moving line heat source. Reference [9] has determined thermal stresses on thin rectangular plate by integral transform with internal moving point heat source. Reference [10] determines temperature distribution, displacement, and thermal stresses of a thin circular plate due to uniform internal energy generation using Hankel transform technique graphically.

Integral transform technique is a powerful tool to solve various new general purpose numerical methods and can be applied to any multidimensional problem to get an approximate solution. This is the easiest way to find parameters like variation of temperature, and so forth. This method is better than other methods.

Attempt is made to determine effective solution and study of thermal stresses in a thin rectangular plate with internally moving heat point source.

Present paper elaborates on determination of temperature and thermal stresses in a thin rectangular plate defined as $0 \leq x \leq a, 0 \leq y \leq b$, and $-h \leq z \leq h$ where $h<b<a$ and $h$ is thickness which is very small. Using integral transform technique the governing heat conduction equation is solved. Results are obtained in the form of infinite series. It has been computed numerically and graphically. 


\section{Formulation of the Problem}

We consider three-dimensional thin rectangular plate under steady state temperature defined in region $R: 0 \leq x \leq a$, $0 \leq y \leq b$, and $-h \leq z \leq h$, where $h<b<a$ and $h$ is thickness which is very small. The plate is subjected to the motion of moving point heat source at the point $\left(x^{\prime}, 0,0\right)$. Under these realistic prescribed conditions, temperature and thermal stresses in a thin rectangular plate are required to be determined.

The temperature distribution of the rectangular plate defined in [11] is given by

$$
\frac{\partial^{2} T}{\partial x^{2}}+\frac{\partial^{2} T}{\partial y^{2}}+\frac{\partial^{2} T}{\partial z^{2}}+\frac{g}{k}=\frac{1}{\alpha} \frac{\partial T}{\partial t},
$$

where $k$ is thermal conductivity and $\alpha$ is thermal diffusivity of the material of the plate.

Consider an instantaneous moving heat source at point $\left(x^{\prime}, 0,0\right)$ and release its heat spontaneously at time $t^{\prime}$. Such volumetric moving heat source in rectangular coordinates is given by

$$
g(x, y, z, t)=g_{p}^{i} \delta\left(x-x^{\prime}\right) \delta(y) \delta(z) \delta\left(t-t^{\prime}\right),
$$

where $g_{p}^{i}$ is instantaneous point heat source.

Hence (1) becomes

$$
\begin{aligned}
& \frac{\partial^{2} T}{\partial x^{2}}+\frac{\partial^{2} T}{\partial y^{2}}+\frac{\partial^{2} T}{\partial z^{2}}+\frac{1}{k} g_{p}^{i} \delta\left(x-x^{\prime}\right) \delta(y) \delta(z) \\
& =\frac{1}{\alpha} \frac{\partial T}{\partial t} .
\end{aligned}
$$

Initial and boundary conditions are given by

$$
\begin{aligned}
{[T]_{t=0} } & =0, \\
{[T]_{x=0} } & =F_{1}(y, z, t), \\
{[T]_{x=a} } & =F_{2}(y, z, t), \\
{\left[\frac{\partial T}{\partial y}\right]_{y=-b} } & =F_{3}(x, z, t), \\
{\left[\frac{\partial T}{\partial y}\right]_{y=b} } & =F_{4}(x, z, t), \\
{\left[T+k_{1} \frac{\partial T}{\partial z}\right]_{z=-h} } & =F_{5}(x, y, t), \\
{\left[T+k_{2} \frac{\partial T}{\partial z}\right]_{z=h} } & =F_{6}(x, y, t) .
\end{aligned}
$$

Thermal stress function $\chi$ is $\chi=\chi_{c}+\chi_{p}$, where $\chi_{c}$ complementary function is and $\chi_{p}$ is particular integral. $\chi_{c}$ and $\chi_{p}$ are governed by equations,

$$
\begin{aligned}
& \left(\frac{\partial^{2}}{\partial x^{2}}+\frac{\partial^{2}}{\partial y^{2}}\right)^{2} \chi_{c}=0 \\
& \left(\frac{\partial^{2}}{\partial x^{2}}+\frac{\partial^{2}}{\partial y^{2}}\right)^{2} \chi_{p}=-\alpha E \Gamma .
\end{aligned}
$$

Since plate is thin, $z$ is negligible and $\Gamma=T-T_{0}$, where $T_{0}$ is initial temperature. Components of stress functions [12] are given by

$$
\begin{gathered}
\sigma_{x x}=\frac{\partial^{2} \chi}{\partial y^{2}}, \\
\sigma_{y y}=\frac{\partial^{2} \chi}{\partial x^{2}}, \\
\sigma_{x y}=-\frac{\partial^{2} \chi}{\partial x \partial y}
\end{gathered}
$$

with boundary conditions $\sigma_{y y}=0$ and $\sigma_{x y}=0$ at $y=b$.

Equations (1) to (8) represent the statement of the problem.

\section{Solution of the Problem}

Applying finite Fourier cosine transform, finite Fourier sine transform [13], and Marchi-Fasulo transform [14], using boundary conditions (4), we get

$$
\frac{d \overline{\bar{T}}^{*}}{d t}+\alpha Q \overline{\bar{T}}^{*}=\alpha \emptyset
$$

where $Q=m^{2} \pi^{2} / a^{2}+n^{2} \pi^{2} / a^{2}+a_{l}^{2}$.

$$
\begin{aligned}
\emptyset= & {\left[\frac{m \pi}{a}\left[(-1)^{m+1} F_{2}+F_{1}\right]+(-1)^{n} F_{3}-F_{4}\right.} \\
& +\frac{p_{l}(h)}{\alpha_{1}} F_{5}-\frac{p_{l}(-h)}{\alpha_{2}} F_{6} \\
& \left.+\frac{g_{p}^{i}}{k} \sin \left(\frac{m \pi x^{I}}{a}\right) p_{l}(0) \delta\left(t-t^{I}\right)\right], \\
\overline{\bar{T}}^{*} & =e^{-\alpha \mathrm{Q} t}\left(\int\left\{e^{\alpha \mathrm{Q} t}+\alpha \emptyset\right\} d t-\int \alpha \emptyset d t\right) .
\end{aligned}
$$

Taking inverse Marchi-Fasulo transform [14], finite Fourier sine transform, and finite Fourier cosine transform [13],

$$
\begin{aligned}
T & =\frac{4}{a b} \\
& \cdot \iint \sum_{l, m, n=1}^{\infty} \frac{p_{l}(z)}{\lambda_{l}}\left\{\left[e^{-\alpha \mathrm{Q} t}\left\{\left(\int e^{\alpha \mathrm{Q} t}+\alpha \emptyset\right) d t-\int \alpha \emptyset d t\right\}\right]\right. \\
& \left.\cdot \cos \left(\frac{n \pi y}{b}\right)\right\} \sin \left(\frac{m \pi x}{a}\right) .
\end{aligned}
$$


And $\Gamma=T-T_{0}$,

$$
\begin{aligned}
\Gamma= & \frac{4}{a b} \sum_{l, m, n=0}^{\infty} \frac{p_{l}(z)}{\lambda_{l}}\left\{\left[e^{-\alpha \mathrm{Q} t}\left\{\int\left(e^{\alpha \mathrm{Q} t}+\alpha \emptyset\right) d t-\int \alpha \emptyset d t\right\}\right]\right. \\
& \left.\cdot \cos \left(\frac{n \pi y}{b}\right)\right\} \sin \left(\frac{m \pi x}{a}\right), \\
\chi_{c}= & \sum_{m=1}^{\infty} y\left[c_{1} e^{m \pi y / a}+c_{2} e^{-m \pi y / a}\right] \cos \left(\frac{m \pi x}{a}\right)+y\left[c_{3} e^{m \pi y / a}\right. \\
& \left.+c_{4} e^{-m \pi y / a}\right] \sin \left(\frac{m \pi x}{a}\right), \\
\chi_{p}= & \frac{4 \alpha E a b}{\pi^{2}\left(a^{2} n^{2}+b^{2} m^{2}\right)} \\
& \cdot \sum_{l, m, n=0}^{\infty} \frac{p_{l}(z)}{\lambda_{l}}\left\{\left[e^{-\alpha Q t}\left\{\int\left(e^{\alpha Q t}+\alpha \emptyset\right) d t-\int \alpha \emptyset d t\right\}\right]\right. \\
& \left.\cdot \cos \left(\frac{n \pi y}{b}\right)\right\} \sin \left(\frac{m \pi x}{a}\right), \\
\chi= & \sum_{m=1}^{\infty} y\left[c_{1} e^{m \pi y / a}+c_{2} e^{-m \pi y / a}\right] \cos \left(\frac{m \pi x}{a}\right)+y\left[c_{3} e^{m \pi y / a}\right. \\
& \left.+c_{4} e^{-m \pi y / a}\right] \sin \left(\frac{m \pi x}{a}\right)+\frac{4 \alpha E a b}{\pi^{2}\left(a^{2} n^{2}+b^{2} m^{2}\right)} \\
& \cdot \sum_{l, m, n=0}^{\infty} \frac{p_{l}(z)}{\lambda_{l}}\left\{\left[e^{-\alpha \mathrm{Q} t}\left\{\int\left(e^{\alpha \mathrm{Q} t}+\alpha \emptyset\right) d t-\int \alpha \emptyset d t\right\}\right]\right. \\
& \left.\cdot \cos \left(\frac{n \pi y}{b}\right)\right\} \sin \left(\frac{m \pi x}{a}\right), \\
\emptyset= & {\left[\frac{m \pi}{a}\left[(-1)^{m+1} F_{2}+F_{1}\right]+(-1)^{n} F_{3}-F_{4}-\frac{p_{l}(h)}{\alpha_{1}} F_{5}\right.} \\
& \left.\frac{p_{l}(-h)}{\alpha_{2}} F_{6}+\frac{g_{p}^{i}}{k} \sin \left(\frac{m \pi x^{I}}{a}\right) p_{l}(0) \delta\left(t-t^{I}\right)\right], \\
&
\end{aligned}
$$$$
T=\frac{4}{a b}
$$$$
\cdot \int \sum_{l, m, n=1}^{\infty} \frac{p_{l}(z)}{\lambda_{l}}\left\{\left[e^{-\alpha \mathrm{Q} t}\left\{\int\left(e^{\alpha \mathrm{Q} t}+\alpha \emptyset\right) d t-\int \alpha \emptyset d t\right\}\right]\right.
$$$$
\left.\cdot \cos \left(\frac{n \pi y}{b}\right)\right\} \sin \left(\frac{m \pi x}{a}\right),
$$$$
\Gamma=T-T_{0},
$$$$
\Gamma=\frac{4}{a b}
$$$$
\cdot \iint \sum_{l, m, n=1}^{\infty} \frac{p_{l}(z)}{\lambda_{l}}\left\{\left[e^{-\alpha \mathrm{Q} t}\left\{\int\left(e^{\alpha \mathrm{Q} t}+\alpha \emptyset\right) d t-\int \alpha \emptyset d t\right\}\right]\right.
$$$$
\left.\cdot \cos \left(\frac{n \pi y}{b}\right)\right\} \sin \left(\frac{m \pi x}{a}\right) \text {. }
$$

As we change the values of $l, m$, and $n$ from 1 to $\infty$ we get infinite terms of this solution which is nothing but infinite series.

\section{Determination of Stress Function}

Using (15) in (6)-(8) we get

$$
\begin{aligned}
& \sigma_{x x}=\sum_{m=1}^{\infty}\left\{\left[2\left(\frac{m \pi}{a} c_{1} e^{m \pi y / a}-\frac{m \pi}{a} c_{2} e^{-m \pi y / a}\right)\right.\right. \\
& \left.+y\left(c_{1} e^{m \pi y / a}+c_{2} e^{-m \pi y / a}\right)\right] \cos \left(\frac{m \pi x}{a}\right) \\
& +\left[2\left(\frac{m \pi}{a} c_{3} e^{m \pi y / a}-\frac{m \pi}{a} c_{4} e^{-m \pi y / a}\right)\right. \\
& \left.\left.+y\left(c_{3} e^{m \pi y / a}+c_{4} e^{-m \pi y / a}\right)\right] \sin \left(\frac{m \pi x}{a}\right)\right\} \\
& +\frac{4 \alpha E a b}{\pi^{2}\left(a^{2} n^{2}+b^{2} m^{2}\right)} \\
& \cdot \sum_{l, m, n=0}^{\infty} \frac{p_{l}(z)}{\lambda_{l}}\left\{\left[e^{-\alpha \mathrm{Q} t}\left\{\int\left(e^{\alpha \mathrm{Q} t}+\alpha \emptyset\right) d t-\int \alpha \emptyset d t\right\}\right]\right. \\
& \left.\cdot \cos \left(\frac{n \pi y}{b}\right)\right\} \sin \left(\frac{m \pi x}{a}\right)\left(\frac{-n^{2} \pi^{2}}{b^{2}}\right), \\
& \sigma_{y y}=\frac{-m^{2} \pi^{2} y}{a^{2}}\left\{\left[c_{1} e^{m \pi y / a}+c_{2} e^{-m \pi y / a}\right] \cos \left(\frac{m \pi x}{a}\right)\right. \\
& \left.+\left[c_{3} e^{m \pi y / a}+c_{4} e^{-m \pi y / a}\right] \sin \left(\frac{m \pi x}{a}\right)\right\}+\left(\frac{m^{2} \pi^{2}}{a^{2}}\right) \\
& \cdot \frac{4 \alpha E a b}{\pi^{2}\left(a^{2} n^{2}+b^{2} m^{2}\right)} \\
& \cdot \sum_{l, m, n=0}^{\infty} \frac{p_{l}(z)}{\lambda_{l}}\left\{\left[e^{-\alpha \mathrm{Q} t}\left\{\int\left(e^{\alpha \mathrm{Q} t}+\alpha \emptyset\right) d t-\int \alpha \emptyset d t\right\}\right]\right. \\
& \left.\cdot \cos \left(\frac{n \pi y}{b}\right)\right\} \sin \left(\frac{m \pi x}{a}\right), \\
& \sigma_{x y}=\left\{\left[\left(c_{1} e^{m \pi y / a}+c_{2} e^{-m \pi y / a}\right)+\frac{m \pi y}{a}\left(c_{1} e^{m \pi y / a}\right.\right.\right. \\
& \left.\left.-c_{2} e^{-m \pi y / a}\right)\right]\left(-\frac{m \pi}{a}\right) \sin \left(\frac{m \pi x}{a}\right)-\left[\left(c_{3} e^{m \pi y / a}\right.\right. \\
& \left.\left.\left.+c_{4} e^{-m \pi y / a}\right)+\frac{m \pi y}{a}\left(c_{3} e^{m \pi y / a}-c_{4} e^{-m \pi y / a}\right)\right]\right\} \\
& \cdot\left(-\frac{m \pi}{a}\right) \cos \left(\frac{m \pi x}{a}\right)+\frac{n m \pi^{2}}{a b} \frac{4 \alpha E a b}{\pi^{2}\left(a^{2} n^{2}+b^{2} m^{2}\right)} \\
& \cdot \sum_{l, m, n=0}^{\infty} \frac{p_{l}(z)}{\lambda_{l}}\left\{\left[e^{-\alpha \mathrm{Q} t}\left\{\int\left(e^{\alpha \mathrm{Q} t}+\alpha \emptyset\right) d t-\int \alpha \emptyset d t\right\}\right]\right. \\
& \left.\cdot \sin \left(\frac{n \pi y}{b}\right)\right\} \cos \left(\frac{m \pi x}{a}\right) .
\end{aligned}
$$


Using the boundary conditions $\sigma_{y y}=0$ and $\sigma_{x y}=0$ at $y=b$, we get

$$
\begin{aligned}
& c_{1}=0 \text {, } \\
& c_{2}=0 \text {, } \\
& c_{3}=\frac{2 \alpha E b \varphi(-a+m \pi b)}{\pi^{2}\left(a^{2} n^{2}+b^{2} m^{2}\right)} e^{-m \pi b / a}, \\
& c_{4}=\frac{-2 \alpha E b \varphi(a+m \pi b)}{\pi^{2}\left(a^{2} n^{2}+b^{2} m^{2}\right)} e^{m \pi b / a}, \\
& \sigma_{x x}=\sum_{m=1}^{\infty}\left\{\left[\left(\frac{2 m \pi}{a}+y\right) \frac{2 \alpha E b \varphi(-a+m \pi b)}{\pi^{2}\left(a^{2} n^{2}+b^{2} m^{2}\right)}\right.\right. \\
& \cdot e^{-m \pi b / a} e^{m \pi y / a}+\left(\frac{2 m \pi}{a}-y\right) \frac{2 \alpha E b \varphi(a+m \pi b)}{\pi^{2}\left(a^{2} n^{2}+b^{2} m^{2}\right)} \\
& \left.\left.\cdot e^{m \pi b / a} e^{-m \pi y / a}\right] \sin \left(\frac{m \pi x}{a}\right)\right\}+\frac{4 \alpha E a b}{\pi^{2}\left(a^{2} n^{2}+b^{2} m^{2}\right)} \\
& \text {. } \sum_{l, m, n=0}^{\infty} \frac{p_{l}(z)}{\lambda_{l}}\left\{\left[e^{-\alpha \mathrm{Q} t}\left\{\int\left(e^{\alpha \mathrm{Q} t}+\alpha \emptyset\right) d t-\int \alpha \emptyset d t\right\}\right]\right. \\
& \left.\cdot \cos \left(\frac{n \pi y}{b}\right)\right\} \sin \left(\frac{m \pi x}{a}\right)\left(\frac{n^{2} \pi^{2}}{b^{2}}\right) \text {, } \\
& \sigma_{y y}=\frac{-m^{2} \pi^{2} y}{a^{2}}\left\{\left[\frac{2 \alpha E b \varphi(-a+m \pi b)}{\pi^{2}\left(a^{2} n^{2}+b^{2} m^{2}\right)} e^{-m \pi b / a} e^{m \pi y / a}\right.\right. \\
& \left.\left.-\frac{2 \alpha E b \varphi(a+m \pi b)}{\pi^{2}\left(a^{2} n^{2}+b^{2} m^{2}\right)} e^{m \pi b / a} e^{-m \pi y / a}\right] \sin \left(\frac{m \pi x}{a}\right)\right\} \\
& -\frac{m^{2} \pi^{2}}{a^{2}} \frac{4 \alpha E a b}{\pi^{2}\left(a^{2} n^{2}+b^{2} m^{2}\right)} \\
& \cdot \sum_{l, m, n=0}^{\infty} \frac{p_{l}(z)}{\lambda_{l}}\left\{\left[e^{-\alpha \mathrm{Q} t}\left\{\int\left(e^{\alpha \mathrm{Q} t}+\alpha \emptyset\right) d t-\int \alpha \emptyset d t\right\}\right]\right. \\
& \left.\cdot \cos \left(\frac{n \pi y}{b}\right)\right\} \sin \left(\frac{m \pi x}{a}\right), \\
& \sigma_{x y}=\left\{\left[-\left(1+\frac{m \pi y}{a}\right) \frac{2 \alpha E b \varphi(-a+m \pi b)}{\pi^{2}\left(a^{2} n^{2}+b^{2} m^{2}\right)}\right.\right. \\
& \cdot e^{-m \pi b / a} e^{m \pi y / a}+\left(1-\frac{m \pi y}{a}\right) \frac{2 \alpha E b \varphi(a+m \pi b)}{\pi^{2}\left(a^{2} n^{2}+b^{2} m^{2}\right)} \\
& \left.\left.\cdot e^{m \pi b / a} e^{-m \pi y / a}\right]\left(-\frac{m \pi}{a}\right) \cos \left(\frac{m \pi x}{a}\right)\right\}+\frac{n m \pi^{2}}{a b} \\
& \frac{4 \alpha E a b}{\pi^{2}\left(a^{2} n^{2}+b^{2} m^{2}\right)} \\
& \text {. } \sum_{l, m, n=0}^{\infty} \frac{p_{l}(z)}{\lambda_{l}}\left\{\left[e^{-\alpha \mathrm{Q} t}\left\{\int\left(e^{\alpha \mathrm{Q} t}+\alpha \emptyset\right) d t-\int \alpha \emptyset d t\right\}\right]\right. \\
& \left.\cdot \sin \left(\frac{n \pi y}{b}\right)\right\} \cos \left(\frac{m \pi x}{a}\right) \text {. }
\end{aligned}
$$

\section{Numerical Results}

Let $k=0.5330, \alpha=23.8 \times 10^{-6}, E=0.675 \times 10^{11}$, and $a=$ $5 \mathrm{~cm}, b=1 \mathrm{~cm}, h=0.2 \mathrm{~cm}$, and

$$
\begin{aligned}
& \sigma_{x x}=\sum_{m=1}^{\infty}\left\{\left[\left(\frac{2 \pi}{5}+y\right)\right.\right. \\
& \cdot \frac{2 \times 23.8 \times 10^{-6} \times 0.675 \times 10^{11} \times \varphi(-5+\pi)}{26 \pi^{2}} e^{-\pi / 5} e^{\pi y / 5} \\
& +\left(\frac{2 \pi}{5}-y\right) \frac{2 \times 23.8 \times 10^{-6} \times 0.675 \times 10^{11} \times \varphi(5+\pi)}{26 \pi^{2}} \\
& \left.\left.\cdot e^{\pi / 5} e^{-\pi y / 5}\right] \sin \left(\frac{\pi x}{5}\right)\right\}+\frac{20 \times 23.8 \times 10^{-6} \times 0.675 \times 10^{11}}{26 \pi^{2}} \\
& \cdot \sum_{l, m, n=0}^{\infty} \frac{p_{l}(0.2)}{\lambda_{l}}\left\{\left[e^{-23.8 \times 10^{-6} \mathrm{Q} t}\left\{\int\left(e^{\alpha \mathrm{Q} t}+\alpha \emptyset\right) d t-\int \alpha \emptyset d t\right\}\right]\right. \\
& \cdot \cos (\pi y)\} \sin \left(\frac{\pi x}{a}\right)\left(\frac{n^{2} \pi^{2}}{b^{2}}\right), \\
& \sigma_{y y}=\frac{-\pi^{2} y}{25}\left\{\left[\frac{2 \times 23.8 \times 10^{-6} \times 0.675 \times 10^{11} \times \varphi(-5+\pi)}{26 \pi^{2}}\right.\right. \\
& \cdot e^{-\pi / 5} e^{\pi y / 5}-\frac{2 \times 23.8 \times 10^{-6} \times 0.675 \times 10^{11} \times \varphi(5+\pi)}{26 \pi^{2}} \\
& \left.\left.\cdot e^{\pi / 5} e^{-\pi y / 5}\right] \sin \left(\frac{\pi x}{5}\right)\right\}-\frac{\pi^{2}}{25} \\
& \frac{20 \times 23.8 \times 10^{-6} \times 0.675 \times 10^{11}}{26 \pi^{2}} \frac{2.3562}{15.5485}\{\cos (\pi y)\} \\
& \cdot \sin \left(\frac{\pi x}{5}\right) \\
& \sigma_{x y}=\left\{\left[\left(-\left(1+\frac{\pi y}{5}\right)\right.\right.\right. \\
& \cdot \frac{2 \times 23.8 \times 10^{-6} \times 0.675 \times 10^{11} \times \varphi(-5+\pi)}{26 \pi^{2}} e^{-\pi / 5} e^{\pi y / 5} \\
& -\left(1-\frac{\pi y}{5}\right) \frac{2 \times 23.8 \times 10^{-6} \times 0.675 \times 10^{11} \times \varphi(5+\pi)}{26 \pi^{2}} \\
& \left.\left.\left.\cdot e^{\pi / 5} e^{-\pi y / 5}\right)\right]\left(-\frac{\pi}{5}\right) \cos \left(\frac{\pi x}{5}\right)\right\}+\frac{\pi^{2}}{5} \\
& \frac{20 \times 23.8 \times 10^{-6} \times 0.675 \times 10^{11}}{26 \pi^{2}} \\
& \frac{p_{l}(0.2)}{\lambda_{l}}\left\{\left[e^{-23.8 \times 10^{-6} \mathrm{Q} t}\left\{\int\left(e^{\alpha \mathrm{Q} t}+\alpha \emptyset\right) d t-\int \alpha \emptyset d t\right\}\right]\right. \\
& \cdot \sin (\pi y)\} \cos \left(\frac{\pi x}{5}\right) \text {, } \\
& \varphi=\frac{2.3562}{15.5485} e^{-23.8 \times 10^{-6} \times 12.4912 t}\left\{\int \left(e^{23.8 \times 10^{-6} \times 12.4912 t}+23.8\right.\right. \\
& \left.\times 10^{-6}(-0.9721-1.2639 t)\right) d t-\int 23.8 \\
& \left.\times 10^{-6}((-0.9721-1.2639 t)) d t\right\} \text {, }
\end{aligned}
$$




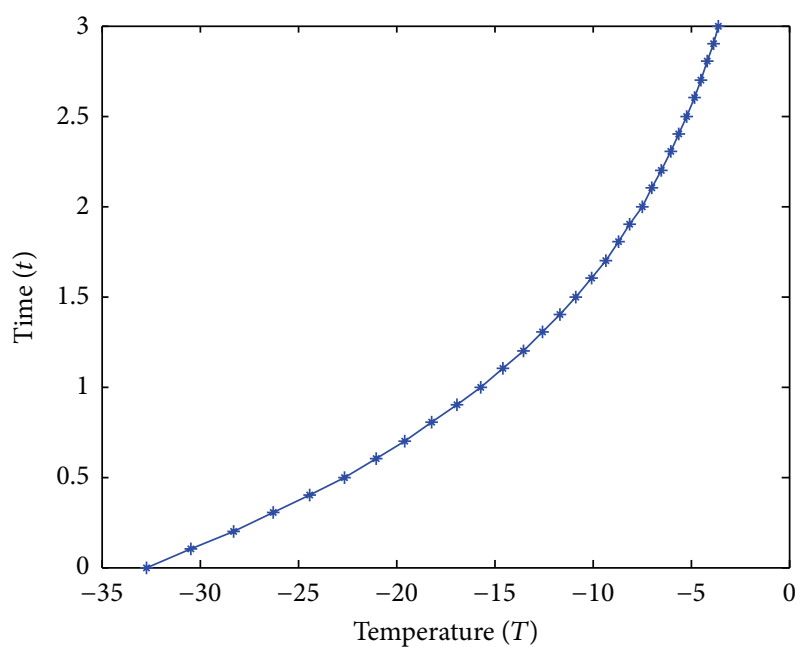

Figure 1: Temperature versus time.

where $x^{I}=1.5, g_{p}^{i}=1, t=1, t^{I}=1.5 \lambda_{l}=15.5485, p_{l}(0.2)=$ $2.3562 \varphi=0.2056 Q=12.4912 \emptyset=-4.5920$, and

$$
\begin{aligned}
T & =29.3083\left\{\left[e^{-2.9729 e-04 t} 4.4206 e^{4.4594 e-04}\right] \cos (\pi y)\right\} \\
& \cdot \sin \left(\frac{\pi x}{5}\right), \\
T= & 40.4676\left\{\left[e^{-0.7369 t}\right] \cos (\pi y)\right\} \sin \left(\frac{\pi x}{5}\right), \\
\sigma_{y y}= & y\left\{\left[10.2767 \times 0.2056 e^{\pi y / 5}+45.0219\right.\right. \\
& \left.\left.\times 0.2056 e^{-\pi y / 5}\right]-5.9124\{\cos (\pi y)\} \sin \left(\frac{\pi x}{5}\right)\right\}, \\
\sigma_{x y} & =\left[(-231.8346+145.6616 y) e^{\pi y / 5}+(71.6544\right. \\
& \left.-45.0205 y) e^{-\pi y / 5}+55.5858\{\sin (\pi y)\}\right] \cos \left(\frac{\pi x}{5}\right), \\
\sigma_{x x} & =\left\{\left[(-463.7527+-369.0536 y) e^{\pi y / 5}\right.\right. \\
& \left.+(143.3047-114.0416 y) e^{-\pi y / 5}+277.9289\right] \\
& \left.\cdot \sin \left(\frac{\pi x}{5}\right)\right\} .
\end{aligned}
$$

\section{Graphical Interpretation}

See Figures 1-7.

\section{Discussion}

In this article the three-dimensional nonhomogeneous heat conduction issue in a thin rectangular plate is studied. We did numerical computations for a thin rectangular plate made up of aluminum. The heat source $g(x, y, z, t)$ is an instantaneous point heat source of strength $g_{i}$. The thermoelastic behavior is examined such as temperature and thermal stresses.

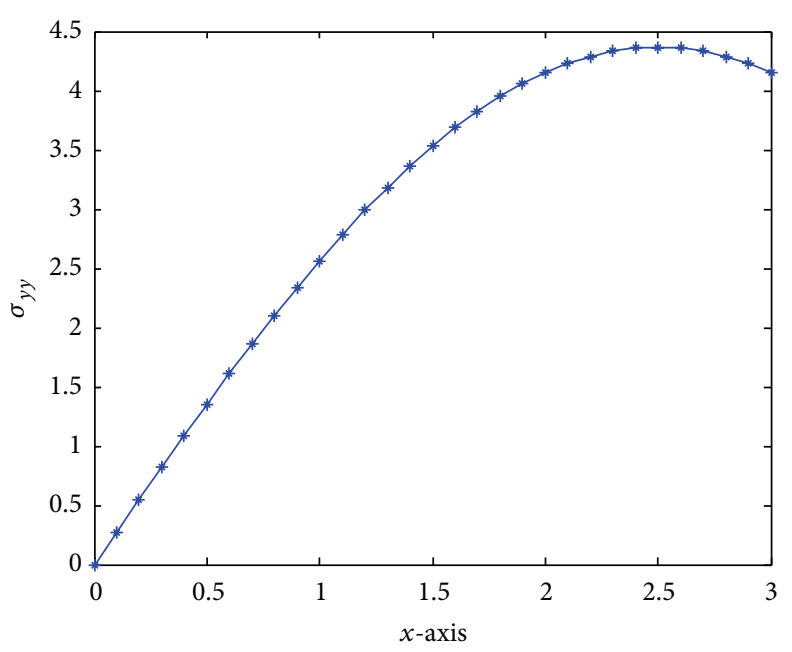

FIGURE 2: $\sigma_{y y}$ versus $x$.

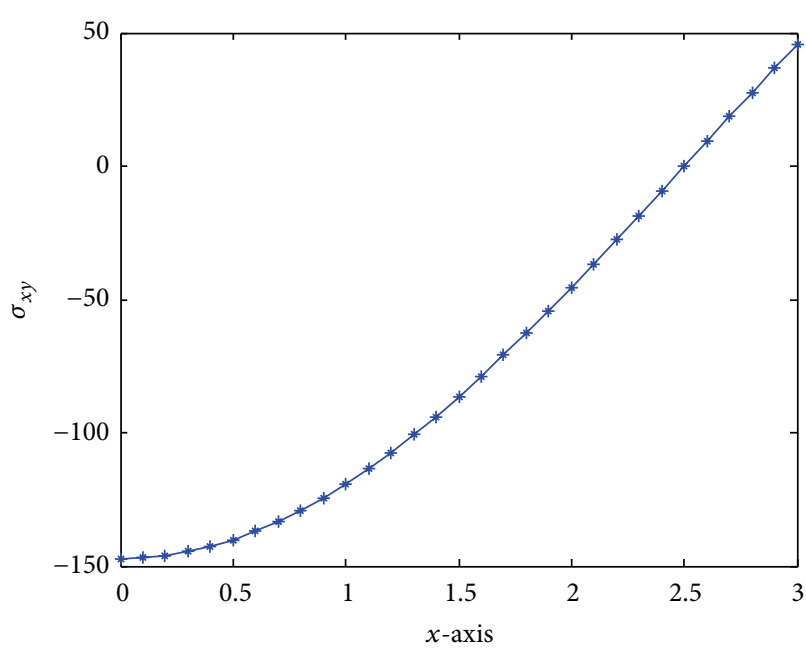

FigURE 3: $x$ versus $\sigma_{x y}$.

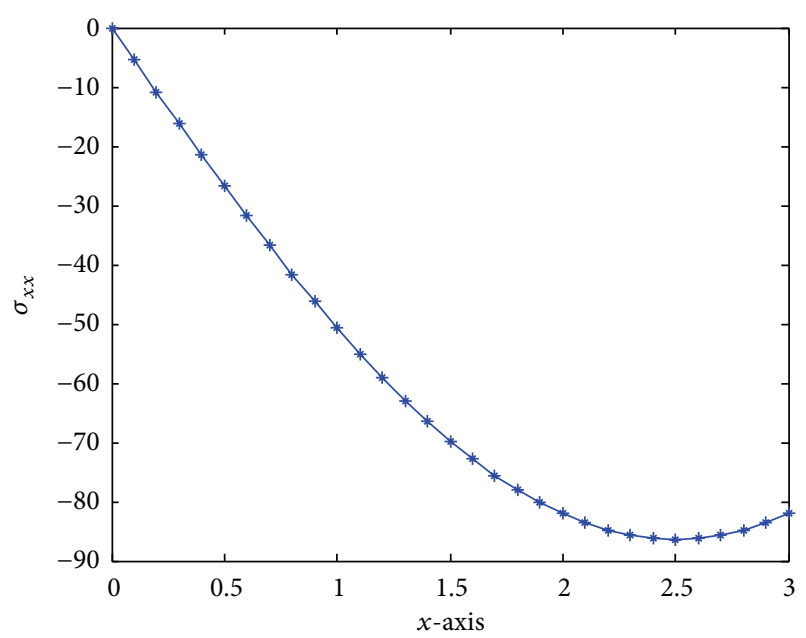

FIGURE 4: $x$ versus $\sigma_{x x}$. 


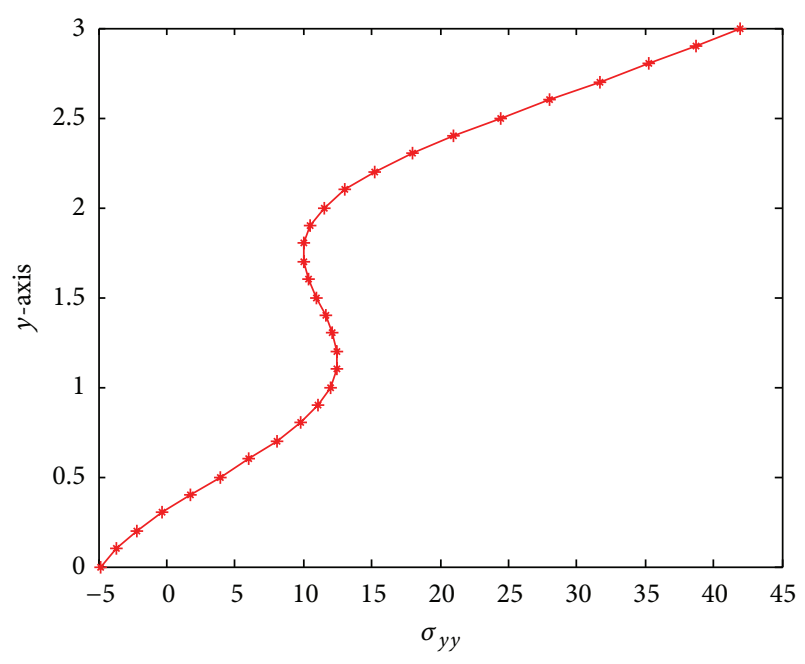

Figure 5: $\sigma_{y y}$ versus $y$.

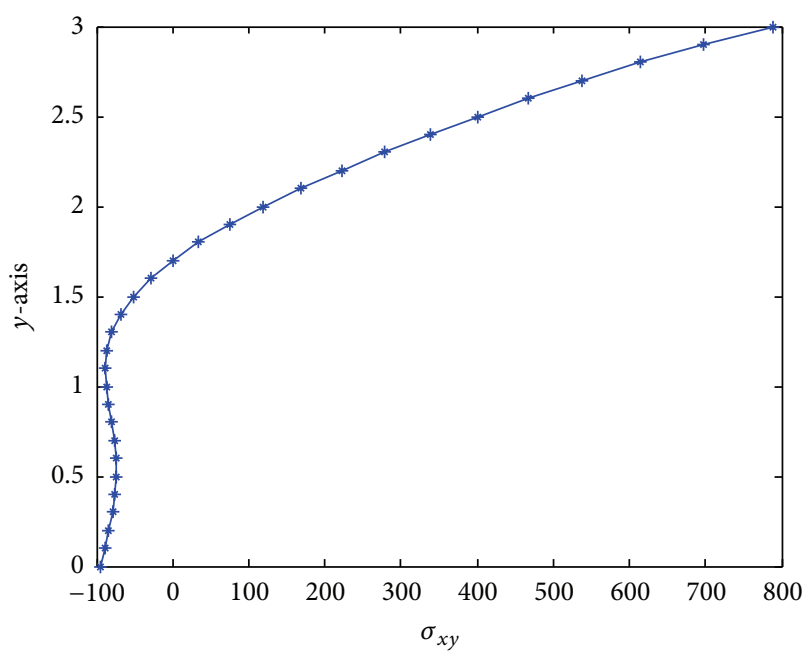

FiguRe 6: $\sigma_{x y}$ versus $y$.

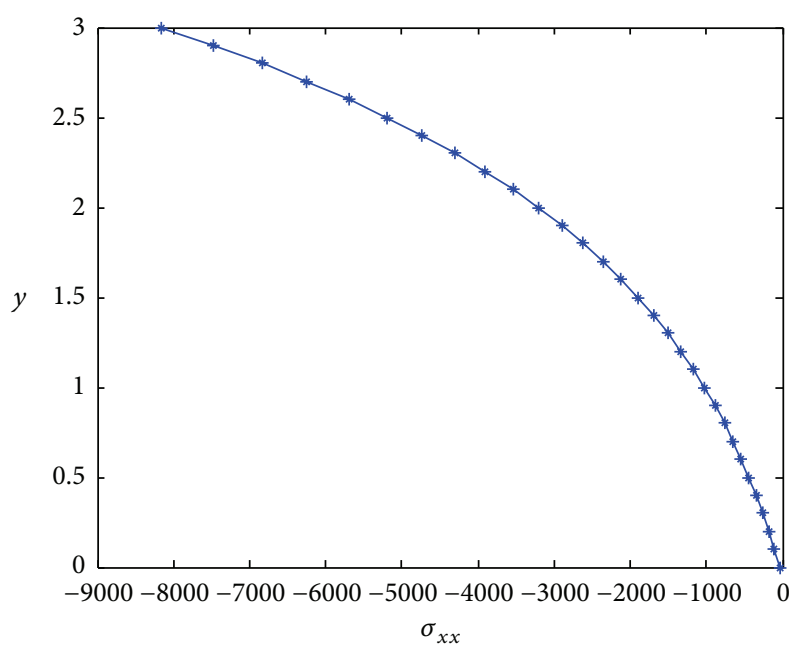

FiguRe 7: $\sigma_{x x}$ versus $y$.
From Figure 1, it is found that at first when time is zero, temperature is shrinking. But as time increases temperature develops as much as precise restriction and it turns out to be regular.

From Figure 2, interatomic distance grew to be extensive up to precise value of $x$ after specific value of $x$ interatomic distance in a plate takes its function as it is. When we provide temperature to aluminium plate, initially atoms in a plate get disturbed; that is, short stress increases along $x$-axis. Thermal stress increases initially, but it is observed that it remains constant and again slightly decreases.

From Figure 3, as temperature rises molecule begins to vibrate more rapidly and push away from one another to increase separation between the atoms that cause expansion in atoms. In a plate, position of atoms gets separated along $x$-axis. Thermal stress of material changes from minimum of $x$ to maximum, that is, variation observed along both $x$-axis and $y$-axis.

From Figure 4, initially when $x$ is zero stress is zero; that is, in a plate interatomic constitution is constant but when we change the worth of $x$ atomic distance gets compressed and at $x=2.5$ it turns into extra compression and again interatomic distance slowly separated. Then it gets its customary position.

From Figure 5, at first interatomic distance could be much closed as altering the worth of $y$ that distance grew to be vast; that is, stress rises.

From Figure 6, stress alongside $y$-axis atomic structure in a plate is rapidly changing its positions as we change the value of $y$.

From Figure 7, it shows that originally at $y=0$ stress is incredibly minimum suggesting that interatomic distance is compressed as value of $y$ changes; it comes to its original interatomic distance so that it acquires its original position.

\section{Conclusion}

In this paper we carried out the nonhomogeneous thermoelastic problem solved using integral transform techniques numerically. Results are obtained dependent on values of $l$, $m$, and $n$ which vary from 1 to $\infty$. Hence variation of heat by moving heat sources in a body changes infinitely. From graphical study when a body is provided with heat, it affects it in all directions. Hence material shows expansion along $x$ axis, $y$-axis, and $z$-axis, respectively. We conclude that if time increases temperature will also increase. Interatomic distance became wide along $y$-axis. As thin rectangular plate subjected to point heat source which changes its place along $x$-axis, interatomic distance became narrow.

The outcomes got here basically applicable in engineering problems, especially for industrial machines subjected to the heating, such as the main shaft of a machine, turbines, the roll of rolling mill, and practical applications in aircraft structures. 


\section{Competing Interests}

The authors declare that there is no conflict of interests regarding the publication of this paper.

\section{References}

[1] J. L. Nowinski, Theory of Thermoelasticity with Application, Sijthoff \& Noordhoff, Alphen Aan Den Rijn, The Netherlands, 1978.

[2] W. Nowacki, "The state of stresses in a thick circular plate due to temperature field," Bulletin of the Polish Academy of Sciences Technical Science, vol. 5, article 227, 1957.

[3] B. A. Boley and J. H. Weiner, Theory of Thermal Stresses, John Wiley \& Sons, New York, NY, USA, 1960.

[4] S. K. Roy Choudhary, "A note of quasi static stress in a thin circular plate due to transient temperature applied along the circumference of a circle over the upper face," Bulletin L'Académie Polonaise des Science, Série des Sciences Mathématiques, vol. 20, pp. 20-21, 1972.

[5] N. W. Khobragade and P. C. Wankhede, "An inverse unsteady state thermoelastic problem of a thin rectangular plate," The Journal of Indian Academy of Mathematics, vol. 25, no. 2, 2003.

[6] K. R. Gaikwad and K. P. Ghadle, "Quasi-static thermoelastic problem of an infinitely long circular cylinder," Journal of the Korean Society for Industrial and Applied Mathematics, vol. 14, no. 3, pp. 141-149, 2010.

[7] V. B. Patil, B. R. Ahirrao, and N. W. Khobragade, "Thermal stresses of semi infinite Rectangular slab with internal heat source," IOSR Journals of Mathematics, vol. 8, no. 6, pp. 57-61, 2013.

[8] D. T. Solanke and M. H. Durge, "Quasi static thermal stresses in thin rectangular plate with internal moving line heat source," Science Park Research Journal, vol. 1, no. 44, pp. 1-5, 2014.

[9] M. S. Thakare, C. S. Sutar, and N. W. Khobragade, "Thermal stresses of a thin rectangular plate with internal moving heat source," International Journal of Engineering and Innovative Technology, vol. 4, no. 9, 2015.

[10] K. R. Gaikwad, "Two-dimensional steady-state temperature distribution of a thin circular plate due to uniform internal energy generation," Cogent Mathematics, vol. 3, no. 1, Article ID 1135720, 2016.

[11] N. M. Ozisik, Boundary Value Problem of Heat Conduction, Dover, Mineola, NY, USA, 1968.

[12] N. Noda, R. B. Hetnarski, and Y. Tanigawa, Thermal Stresses, Lastran, 2nd edition, 2002.

[13] I. N. Sneddon, The Use of Integral Transform, McGraw Hill, New York, NY, USA, 1972.

[14] E. Marchi and A. Fasulo, "Heat conduction in sectors of hollow cylinders with radiation," Atti della Accademia delle Scienze di Torino, no. 1, pp. 373-382, 1967. 

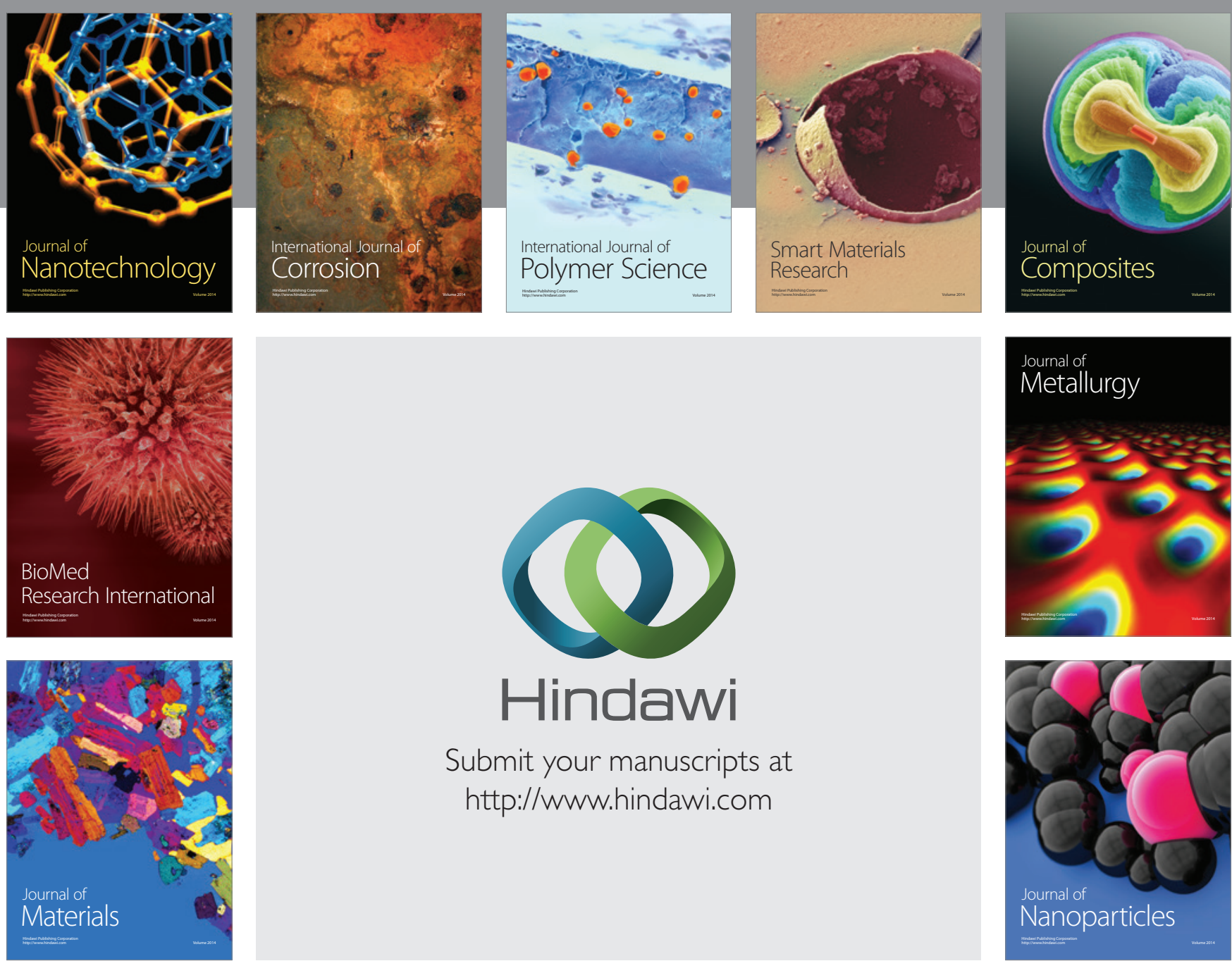

\section{Hindawi}

Submit your manuscripts at

http://www.hindawi.com

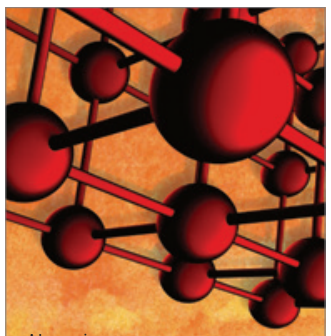

Materials Science and Engineering
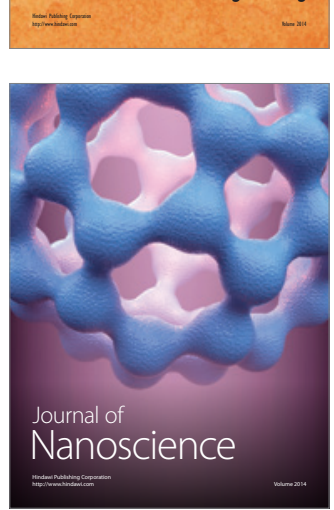
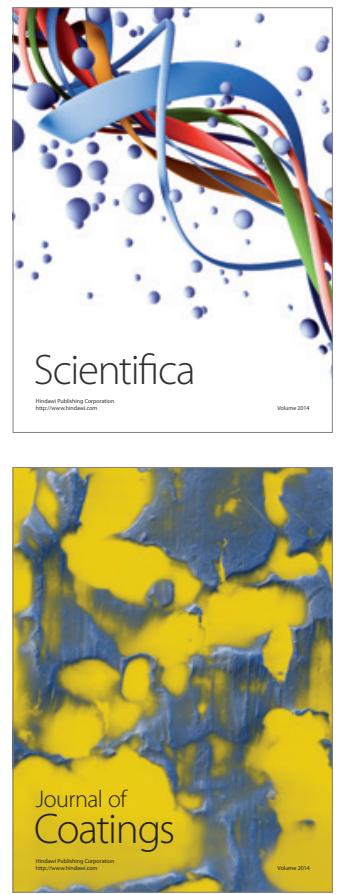
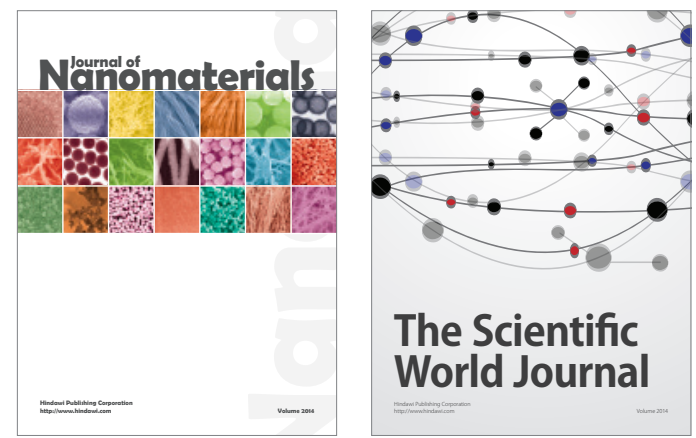

The Scientific World Journal
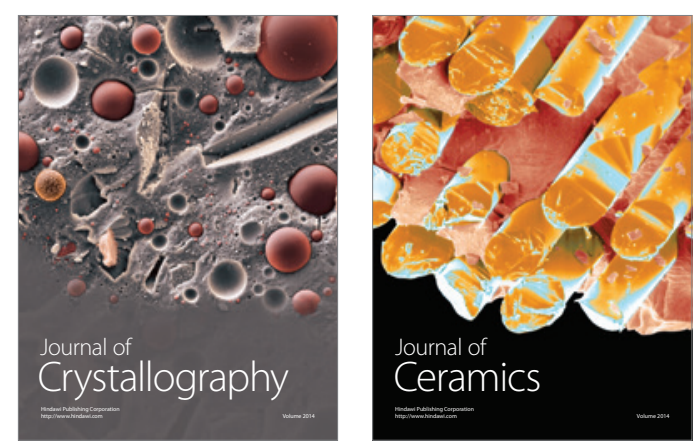
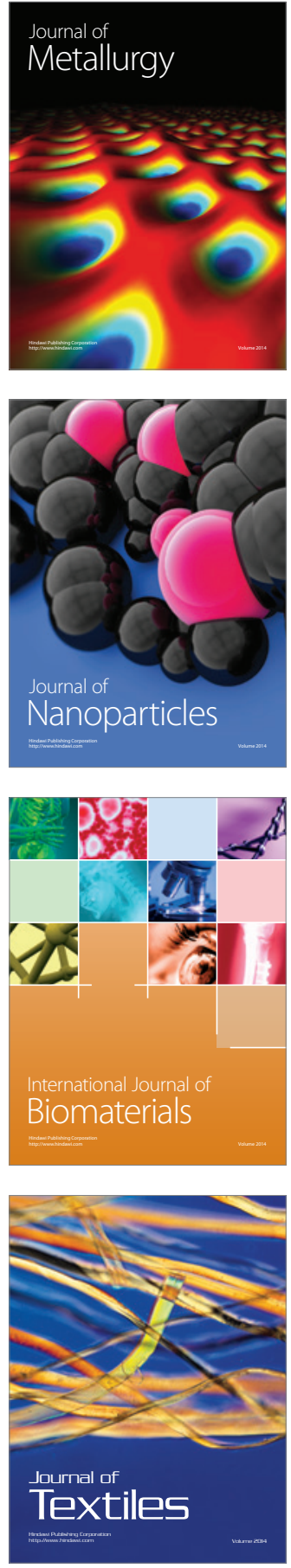